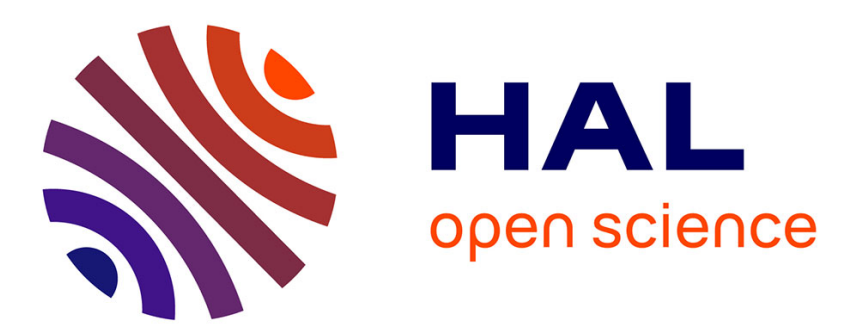

\title{
Towards efficient disaster management: 5G and Device to Device communication
}

\author{
Majed Haddad, Priyanka Rawat, Eitan Altman
}

\section{To cite this version:}

Majed Haddad, Priyanka Rawat, Eitan Altman. Towards efficient disaster management: 5G and Device to Device communication. 2015 2nd International Conference on Information and Communication Technologies for Disaster Management (ICT-DM), Nov 2015, Rennes, France. pp.79-87, 10.1109/ICT-DM.2015.7402056 . hal-03485192

\section{HAL Id: hal-03485192 \\ https://hal.science/hal-03485192}

Submitted on 17 Dec 2021

HAL is a multi-disciplinary open access archive for the deposit and dissemination of scientific research documents, whether they are published or not. The documents may come from teaching and research institutions in France or abroad, or from public or private research centers.
L'archive ouverte pluridisciplinaire HAL, est destinée au dépôt et à la diffusion de documents scientifiques de niveau recherche, publiés ou non, émanant des établissements d'enseignement et de recherche français ou étrangers, des laboratoires publics ou privés. 


\section{Towards efficient disaster management: $5 \mathrm{G}$ and Device to Device communication}

\author{
Priyanka Rawat \\ CERI/LIA, University of Avignon, France \\ priyanka.rawat@univ-avignon.fr
}

\author{
Majed Haddad \\ CERI/LIA, University of Avignon, France \\ Majed.Haddad@univ-avignon.fr
}

\author{
Eitan Altman \\ INRIA Sophia Antipolis, France \\ Eitan.Altman@sophia.inria.fr
}

\begin{abstract}
Recent events of multiple earthquakes in Nepal and resulting loss of life and resources bring our attention to the ever growing significance of disaster management, especially in the context of large scale nature disasters such as earthquake and Tsunami. In this paper, we focus on how disaster management can benefit from recent advances in wireless communication technologies and protocols, especially mobile technologies and devices. The paper provides an overview of how the new generation of telecommunications and technologies such as 5G, Device to Device, 4G/LTE, and software defined radio can improve the potential of disaster management networks. Our survey is different from existing surveys in that we focus on recent advances and ongoing research directions in disaster management with the focus being on the use of ubiquitous mobile devices and applications.
\end{abstract}

Keywords: Disaster communication, 5G, Device to Device communication, LTE, Mobile phone disaster mode, Survey.

\section{INTRODUCTION}

Recent events of multiple earthquakes in Nepal and resulting loss of life and resources bring our attention to the ever growing significance of disaster management especially in the context of large scale nature disasters such as earthquake and Tsunami. The earthquake hit Nepal on 25th April causing the death of over 8000 people and rendering over 250,000 people homeless and injured as well as large scale destruction of the basic infrastructure and century old heritage and temples [1]. This reminded us how in the absence of a good disaster infrastructure in place, the people were left scrambling to respond. On one hand, this evokes discussion on how an efficient disaster infrastructure in place could have helped in preventing and minimizing the loss of lives and property by alerting the people about the impending disaster; considering it is almost impossible to predict an earthquake. On the other hand, it was interesting to see that in the immediate aftermath of the earthquake, what technologies and tools were proved to be useful to the people affected by the disaster as well as the rescuers and authorities. Immediately after the first earthquake, there were messages of safety reports on social media. Facebook activated its Safety Check feature to enable people to give quick safety status updates to their family and friends during the disaster [2]. Popular social media tools like Facebook and Twitter were flooded with messages for requests for help and after 2 days Facebook and Twitter exploded. It was observed that on the ground, local people had little capacity to gather and arrange the large amounts of information coming in and this led to confusion due to the limitations of the cellular networks to handle the traffic. Moreover, the utility of social media is usually limited outside urban population centres as for example, only $40 \%$ of Nepal is online [1]. Nevertheless, in the lack of Internet connectivity, a person in rural area can send text to someone in urban area, who can then upload the message on social media and then, from there the message can be picked up by a disaster relief team. However, the impact of such efforts are very limited on the ground.

In recent years, the focus of research in disaster management has been on designing systems that allow affected people in disaster situations to communicate with their family as well as rescue teams [3]-[6]. However, most of the present disaster management systems rely on an existing network infrastructure. Though there are some studies [7], [8] examining the use of Bluetooth (BT) or Wi-Fi in Ad Hoc mode to provide communication platforms that are independent from existing network infrastructure, they usually rely on special hardware or custom modified mobile systems.

In this paper, we focus on how disaster management can benefit from recent advances in wireless communication technologies and protocols, especially mobile technologies and devices. The paper surveys various potential emerging wireless communication technologies such as 5G, D2D (Device to Device), 4G/LTE, and software defined radio for disaster situations. In particular, we focus on the use of ubiquitous mobile devices and applications in disaster situations. It is interesting to note that according to Cisco forecasts the number of mobile-connected devices exceeded world's population in 2014 and there will be 11.5 billion mobile-connected devices by 2019 [9]. With the ever growing number of mobile devices, the current ubiquity of mobile devices make them an integral component of disaster management. The paper is organized as follows. Section II describes recent advances in disaster management systems and approaches along with some limitations of the existing approaches. Section III provides an overview of disaster management systems and networks. Section IV presents several potential emerging wireless communication technologies for disaster situations. Next, in section $\mathrm{V}$ we discuss solutions for disaster management that focus on the use of mobile phone. Section VI identifies open research challenges and finally, we conclude in section VII.

\section{RECENT ADVANCES}

Recent developments in wireless communication, sensor technology (RFID), and robotics have led to techniques and approaches that can be effectively used to locate and rescue victims during disaster situations. As a result, in last few 
years, we have witnessed some progress in research related to disaster management. Many systems that are currently in place for enabling people to communicate during emergency situation include M-Urgency and SafeCity [3]-[6]. M-Urgency [3] enables iOS or Android users to stream live reports (video as well as audio) over the cellular network to a local publicsafety answering point. It also provides real-time position through GPS or Wi-Fi fingerprint to ensure an appropriate help. SafeCity [5] enables receiving live mobile video stream of crimes and crises situations. However, in disaster scenario, existing emergency response systems require the network infrastructure to be still operable.

Some existing technologies overcome this problem by allowing to create ad-hoc communication networks during a crisis, for example, OpenGarden [10] offers access to online services to offline users by allowing participating devices tether via low-power Bluetooth radio to the device that can connect to the Internet. Wi-Fi Direct enables ad-hoc communication, however its main mechanisms do not support legacy Wi-Fi devices, but only support Wi-Fi Direct certified devices [11]. Work in [12], [13] address the issue of providing communication channels in disaster situations where network infrastructure has become disrupted. The Twimight [7] application that runs on Android devices enables communication between Twitter users in proximity via BT despite the disruptions to the fixed communication network. Twimight builds on Bluetooth, as in comparison to Wi-Fi, its operation is more energy efficient. However, BT has limited range and a cumbersome pairing process. Work in [12] supports the discovery and connection of wireless devices by $\mathrm{Wi}-\mathrm{Fi}$ in ad hoc mode to allow for opportunistic communication. A device advertises its presence by broadcasting beacons carrying information (e.g., device ID) in the SSID field, and thus enables detecting several devices at the same time since one device gets various beacons from different devices without setting up a connection to them. This approach is relatively energy efficient with acceptable connection speed among peered devices, however it does not run on commercial smartphones as it uses Wi-Fi in Ad Hoc mode. Whereas Wifi-Opp [13] uses Wi-Fi in Infrastructure mode and enables opportunistic networking without root access on Android mobiles.

We can identify some limitations of the existing disaster management systems and approaches: (i) usually rely on existing network infrastructure (mobile broadband, Wi-Fi hotspots), that is prone to disruption in disaster e.g., as a result of overloading, (ii) systems using Bluetooth are not adequate for rapid communications among devices, because of the configuration process required for Bluetooth pairing mechanism, (iii) $\mathrm{Wi}-\mathrm{Fi}$ in Ad Hoc mode need devices to run as root (Android).

\section{OVERVIEW OF DISASTER MANAGEMENT SYSTEMS}

In the event of a disaster (e.g., an earthquake), the utmost priority is to provide relief operation to the affected people on the ground. An efficient relief operation involves establishing (re-establishing) of communication infrastructure that could be damaged, performing search and rescue operation, and providing basic services like food, water, shelter and medical help to the victims.
TABLE I. DISASTER MANAGEMENT PHASES.

\begin{tabular}{l|l}
\hline Pre-disaster phase & Post-disaster phase \\
\hline Disaster mitigation & $\begin{array}{l}\text { Disaster recovery } \\
\text { and relief operation }\end{array}$ \\
Alert/Warning systems & $\begin{array}{l}\text { Rescuing affected people } \\
\text { Setting up communication } \\
\text { Mobile phone disaster mode }\end{array}$ \\
Disaster Apps & \begin{tabular}{l} 
Social Media e.g., Twitter \\
\hline
\end{tabular}
\end{tabular}

TABLE II. REQUIREMENTS FOR A DISASTER MANAGEMENT SYSTEM.

\begin{tabular}{|c|c|}
\hline Requirements & Related features (description) \\
\hline Fast response & $\begin{array}{l}\text { Rapid deployment of disaster recovery infrastructure } \\
\text { Must be operational quickly to meet victims' needs }\end{array}$ \\
\hline $\begin{array}{l}\text { Life expectancy of } \\
\text { the network or system }\end{array}$ & $\begin{array}{l}\text { Must operate effectively until the completion of } \\
\text { rescue operation, or until (possibly) restoration of } \\
\text { normal infrastructure }\end{array}$ \\
\hline Interoperability & $\begin{array}{l}\text { Must be able to work or interoperate with existing } \\
\text { infrastructure }\end{array}$ \\
\hline Tariff-free operation & $\begin{array}{l}\text { Free or low cost availability to victims } \\
\text { enabling the use of disaster mode applications } \\
\text { e.g., voice-based apps to connect with others }\end{array}$ \\
\hline Network coverage & $\begin{array}{l}\text { Enable interconnecting the damaged communication } \\
\text { infrastructure in different disaster zones quickly } \\
\text { Solution for providing network coverage in disaster } \\
\text { zone with } 1 \text { network or a cluster of networks that } \\
\text { can be interconnected to allow communication across } \\
\text { the disaster zone }\end{array}$ \\
\hline $\begin{array}{l}\text { Support for heterog- } \\
\text {-eneous traffic types }\end{array}$ & $\begin{array}{l}\text { Support for different traffic types } \\
\text { e.g., voice, data or video apps }\end{array}$ \\
\hline Network capacity & $\begin{array}{l}\text { Enough capacity to handle communication sessions } \\
\text { (traffic) generated by victims as well as rescue teams }\end{array}$ \\
\hline $\begin{array}{l}\text { Easy to use and } \\
\text { equipment cost }\end{array}$ & $\begin{array}{l}\text { Low cost and simple to use equipments e.g., mobile } \\
\text { devices (phones) equipped with disaster apps enabling } \\
\text { victims/rescue teams to access wireless service }\end{array}$ \\
\hline $\begin{array}{l}\text { Outdoor and } \\
\text { indoor scenarios } \\
\text { High precision for }\end{array}$ & $\begin{array}{l}\text { Must support both indoor and outdoor disaster } \\
\text { scenarios in a seamless manner }\end{array}$ \\
\hline $\begin{array}{l}\text { localisation and } \\
\text { search operation }\end{array}$ & Must provide reasonably accurate location of victims \\
\hline
\end{tabular}

We can classify disaster management into two phases; predisaster phase and post-disaster phase as shown in Table I [14]. The pre-disaster phase involves disaster mitigation whereas in the post-disaster phase the focus is on disaster recovery and relief operation in the affected areas. The post-disaster phase covers setting up of communication infrastructure, locating and rescuing the victims, providing basic needs including medical help. Pre-disaster phase is as important as the post-disaster phase, because an effective approach in pre-disaster phase leads to an efficient disaster management in the post-disaster phase.

An effective post-disaster phase depends on the technologies (and tools) available that can be used by the affected people on the ground as well as the professional first responders. The affected people should be able to use the available technologies as fast as possible to communicate their location and whereabouts to the first responders. We are interested in investigating technologies and protocols that enable a rapid and efficient rescue operation in a disaster scenario. Table II highlights the key requirements that should be met by a disaster management system [14]. In this paper, we assume disaster management system based on wireless networks and therefore, the requirements are specific to wireless communication networks. 


\section{POTENTIAL FUTURE WIRELESS COMMUNICATION TECHNOLOGIES FOR DISASTER SCENARIO}

As it was observed in Nepal, due to the earthquakes most of the existing networks were completely destroyed in the disaster affected areas and this rendered local people as well as first responders helpless and large number of victims trapped in the disaster zones for several days. Such large scale disasters highlight the necessity of an efficient disaster management system that can operate in pre- and post-disaster phases. The basic requirements for a disaster management network include autonomicity, interoperability with the existing communication infrastructure (existing in the pre- and post-disaster phase), easily deployable and operable allowing rapid relief operation.

In view of these requirements, wireless networks are preferred for disaster relief operations as usually wireless networks do not need any pre-existing infrastructure to be set up and are easy to operate. Over the years, many architectural solutions and protocols have been proposed and developed that use combinations of existing (wireless communication) technologies for disaster relief operations. Disaster management systems have evolved over the years and with the occurrence of many large scale disaster events like earthquake, Tsunami in the last 10 years, there is much interest in new advanced technologies (solutions and approaches) for disaster scenarios. In this section, we describe potential emerging wireless technologies for disaster scenarios.

\section{A. Role of $5 G$ in disaster management}

The 4G cellular technology LTE and LTE-A offer an unprecedented quality of experience (QoE) for the users. In the context of disaster management, there has been much focus on enhancing the features of LTE-A (in the release 12 of 3GPP) to meet the requirements of public safety and disaster situation communications [15]. However, in recent years, there has been an exponential growth in mobile data traffic and thus, the huge traffic volumes generated by mobile users is creating the need for the next generation of a full IP cellular technology, referred to as 5G [16]. The upcoming $5 \mathrm{G}$ systems are envisioned to have the crucial capabilities such as network flexibility, (re)configuration and resilience and therefore, expected to play a key role in improving disaster situation communications. Furthermore, in 5G, network will provide media independent handover (IEEE 802.21 support) allowing seamless handoff between various available networks thereby enabling disaster communication without any disruption. 5G networks are not only expected to provide much faster transmission throughput, but also support the emerging use-cases related to the Internet of Things (IoT), Machine Type Communications (MTC), broadcast-like services and even lifeline communications during natural disasters. These emerging scenarios generate new demands and $5 \mathrm{G}$ will fulfill these demands by adopting new technologies like proximity services (ProSe), through which devices communicate with each other directly instead of relying on base stations (eNodeB) of network operators.

One of the promising technologies that will support the $5 \mathrm{G}$ architecture and is being considered in the future $5 \mathrm{G}$ standard Long Term Evolution (LTE) Release 13 and beyond, is Device-to-Device communications (D2D). D2D can be used

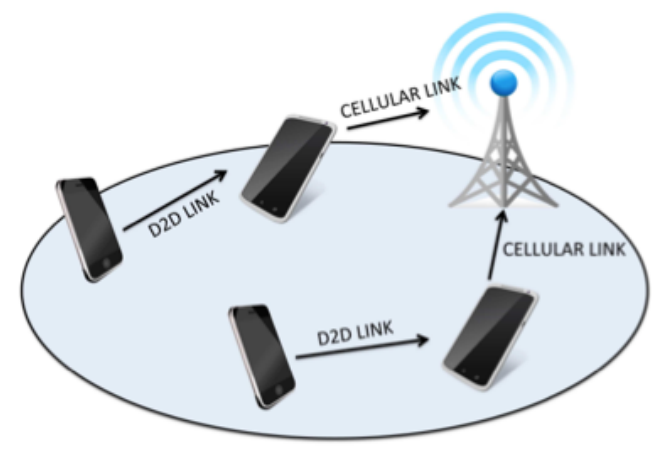

Fig. 1. Device-to-Device based content uploading [17].

for different proximity-based services where the users locate their neighbors and benefit from various services like social apps, and alert messages [17]. D2D can benefit in a disaster scenario (public safety and warning messages) to manage the radio spectrum and energy consumption for providing high QoE and better QoS. In the following subsection, we further describe D2D communications for disaster scenarios.

1) Device to Device (D2D) communications for disaster scenarios: In disaster, the effective use of the radio resources is of extreme importance with the goal of serving a large number of affected people to collect information from different nodes in the disaster zone. In this context, D2D communication will be effective as for example, a D2D-based solution allows an efficient spectrum allocation without adding any further delay in content uploading for the UEs (User Equipments).

D2D communications in wireless network are termed as the direct communication between two devices without the use of the network infrastructure [18]. D2D offers a wide range of advantages from offload cellular traffic, reduced battery consumption, much higher data-rate to novel applications [19]. In particular, D2D communications are of great interest in scenarios, where there is a high density of devices sharing the scarce cellular radio resources, e.g., in public safety and disaster scenarios where the network coverage and the connectivity are limited with the network infrastructure having been damaged. A recent review of key requirements, technology challenges, and solution approaches for enabling D2D to meet the requirements for public protection and disaster relief is presented in [20]. Work in [17] explores the possibility of D2D communication between UEs in proximity to each other to minimize the radio resource needed to upload multimedia content to the base station (eNodeB). The reference scenario is a single cell in a LTE-A system, where multiple UEs aim at uploading some data content to a central server or to the Cloud as shown in Figure 1 [17]. Such a D2D-based solution allows an efficient spectrum allocation without adding any delay in content uploading for the UEs. Authors claim that in comparison to a standard LTE uploading scheme, the proposed D2D-based solution is more effective as it decreases the average number of RBs (Resource Blocks) required in a data uploading process without affecting the standard uploading time given by the LTE system. Moreover, it provides a more effective management of the battery life as compared to the traditional LTE.

Note that the schedulers that are often implemented in 
LTE systems (e.g., best Channel Quality Indicator (BCQI), Proportionally Fair (PF) and Round Robin (RR)) mostly target the best resources utilization for increasing fairness, throughput and bandwidth efficiency and assign them more promptly and faster to the users. However, in disaster scenarios, it is required to design smarter resource scheduling to prevent the loss of connectivity and resulting isolation of first responders located inside buildings or tunnels. This problem is addressed in [21] and different scheduling disciplines in 5G Systems for emergency communications, especially in post-disaster phase are provided. A novel D2D based messaging solution to overcome the UE power limitation issues faced by cellular radio access technologies in disaster situations is presented in [22]. The proposed D2D messaging mechanism has been compared with the default RACH (Random Access Channel) based messaging mechanism. However, in this work the RACH based power consumption is not explicitly modeled and since, for each radio access technology (GSM, UMTS, LTE), the $\mathrm{RACH}$ mechanism is slightly different, further study is needed to evaluate the power consumption. Work in [23] proposes two D2D solutions for content uploading to reduce the uploading time in comparison to the standard LTE transmission. More recently, Nishiyama et al. [24] have developed a prototype (termed as Relay by Smartphone) of D2D relaying smartphone that enables sending out emergency messages from disconnected areas (e.g., mountains, islands) and sharing of information between people gathered in evacuation centres.

\section{B. Long Term Evolution (LTE)}

With 5G technology still in the process of being standardized and far from reaching the markets in near future, LTE will continue to be the choice for disaster and emergency communication networks. According to ABI research forecasts, $5 \mathrm{G}$ subscriber growth would be a bit more muted initially because of the higher complexity of 5G cells and networks [25]. In view of this, LTE is considered as the leading technology for future commercial mobile networks. The adoption of LTE technology enables delivering the increasingly data-intensive applications demanded by the disaster management system [26]. The suitability of LTE for mobile broadband disaster relief operations is discussed in [27]. LTE has been designed to provide a highrate, very low latency IP connectivity service between UE and external IP networks, referred to as Packet Data Networks (PDNs). This IP connectivity service can be utilized by almost any application relying on IP communication, enabling a large number of services to be provided over LTE networks [27].

LTE has the potential to be the next generation wireless technology for disaster management though some issues need to be addressed such as interoperability issue i.e., its ability to co-exist and integrate with the existing infrastructures. Future research should consider designing algorithms for resource management to balance the resources across different dimensions (frequency, time, space, services) within the timing constraints of the disaster management operational scenarios where loss of time in providing the resources or deterioration in the offered services can lead to a higher loss of lives. Sharing of LTE networks could be more effective in a disaster scenario in urban area due to the presence of cellular network infrastructures with decent coverage. In this context, SelfOrganizing Networks (SON) as proposed in [28] can be an efficient solution to optimize network resources, support users' mobility and energy saving, which are all very crucial parameters for a disaster management system.

\section{Software Defined Radio}

While LTE provides a solution to address the lack of broadband connectivity in the disaster network, Software Defined Radio (SDR) technology provides a solution to address the lack of inter-operability in a wireless communication scenario e..g, in military domain. SDR enables a platform to interface and communicate with different communication technologies. One example of an SDR technology based platform namely NAV/COM platform for emergency services is provided in [29]. Work in [29] identifies the main benefits and limitation of SDR platforms for emergency services. In [30], the benefits of SDR technology are discussed through its application to the Public Safety domain. In scenarios where public safety as well as military organizations should engage in the disaster response, one of the main challenges is the presence of interoperability barriers in the disaster area. SDR technology could be used to support various wireless communications technologies on the same radio platform. It is also essential to define a common waveform to support the wireless backbone network.

Though SDR is a promising technology, its potential application in the disaster management requires addressing various issues; for example, (i) Military oriented solutions for SDR equipment are rather costly for disaster applications, (ii) Waveform processing in SDR need significant energy and computing resources that might be a problem for handheld terminals.

\section{Cognitive Radio}

Cognitive Radio is defined as "an intelligent radio that can obtain the knowledge of radio operational environment and established policies and monitor usage patterns and users' needs; to dynamically and autonomously adjust its operational parameters and protocols". The term Cognitive Radio (CR) was coined by Joseph Mitola [31]. The functions that CR should provide include spectrum sensing, spectrum management, spectrum sharing and spectrum mobility [26]. These CR functions enable the flexibility needed in disaster management in affected area where fixed communication infrastructures may be damaged.

In disaster scenarios, emergency responders are increasingly using wireless communications to prevent or respond to emergency incidents. Public safety agencies are using tools like video surveillance cameras and sensors. The radio frequencies allocated for public safety use [32] are getting congested. Moreover, there are interoperability issues due to the use of multiple frequency bands, incompatible radio equipment, and lack of standardization. The existing wireless communication systems' infrastructure will not be able to cope with the future demands of emergency response. Work in [33] focuses on how public safety communications systems can benefit from the CR technology during disaster. The opportunistic spectrum usage provided by CR technology will allow efficient and reliable emergency network transmission. With CR, public safety users can use additional spectrum such as TVWS for daily operation. In addition, public safety agencies can access 


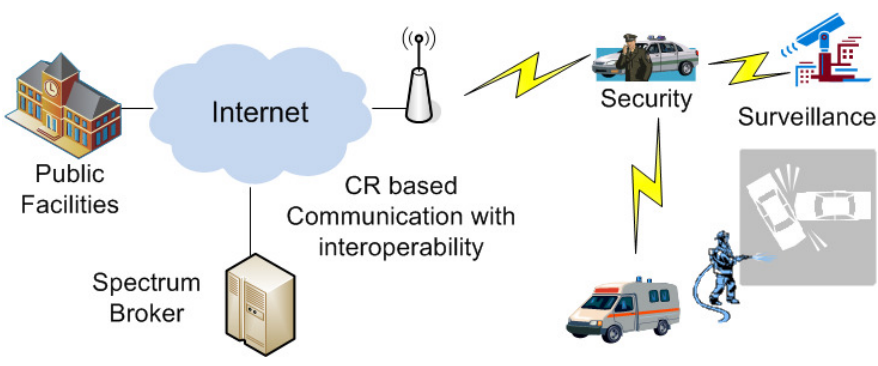

Fig. 2. CR enabled emergency networks.

licensed spectrum and/or commercial networks in $700 \mathrm{Mz}$ (designated by FCC for emergency use) by doing spectrum sharing partnerships with commercial operators, e.g., in areas where public safety wireless network is not available or an effective response to emergency requires more network capacity [33]. Emergency network applications for CR include locating survivors accurately in extreme environments and improving reliability and efficiency of communications. Energy efficiency is significant as emergency rescue operations are usually hampered by low battery life. Work in [34], proposes a CR approach for emergency communication based on SingleCarrier Frequency Division Multiple Access (SC-FDMA), that can enable the emergency terminal to dynamically search for spectrum resources wherever they are available (e.g., not used) in the disaster zone. In [35], the authors propose the application of spectrum pooling for public safety and describes a CR approach through a dynamic policy front end to regulate emergency redistribution of spectrum in case of disaster.

As shown in Figure 2, in a CR-enabled public safety network, location-aware and/or sensing-capable CR devices along with the spectrum coordinator respond to the emergency and coordinate with users in the emergency incident area to ensure that first responders can communicate efficiently [36]. Moreover, CR can improve device interoperability through spectrum agility and interface adaptability, or a network of multiple networks. CR devices can communicate directly with each other by switching to common interface and frequency. Furthermore, CR can facilitate communications for devices operating in different bands and/or having incompatible wireless interfaces. Further research is required to improve the role of CR in emergency networks that include investigating how CR technologies will support priority delivery and routing of content through its own network as well as public networks, thus protecting time-sensitive life-saving information from loss or delay due to network congestion.

\section{E. Indoor position technologies}

In disaster scenarios, Global Navigation Satellite Systems (GNSS) based positioning is used to enhance the coordination of the rescue teams. However, due to the lack of GNSS coverage in indoor environments such as tunnels, buildings, indoor navigation is required in these cases for providing the location services to first time responders. Work in [37] proposes an UltraWideBand (UWB) technology based indoor positioning system for disaster scenario and describes the benefits of UWB technology for indoor positioning. UWB is a radio-frequency signal, that occupies a large bandwidth in the radio-frequency spectrum. UWB ranging based on time of arrival (TOA) can be used for localization. UWB can alleviate multipath with the excess bandwidth available. Moreover, UWB TOA systems are highly accurate thanks to the high time domain resolution [38]. Work in [39] discusses the use of WiFi for ranging in disaster management applications. It presents a prototype of an experimental framework that uses a WiFi network enabling a mobile device to locate its indoor position. A ground-based constellation of pseudo-satellites known as Pseudolites can be deployed in an indoor environment, where GNSS signals from the satellites are feeble to be processed by extremely sensitive GNSS receivers. As described in [40], using Pseudolites in disaster scenario can be effective if they are deployed in disaster zone quickly to provide sufficient coverage and if the issues related to wireless interference generated by pseudolites e.g., to the GNSS satellite signal (outside the indoor area) can be addressed.

In order to make indoor positioning a potential technology for disaster scenario, some of the issues that need to be addressed are; (i) indoor-positioning devices should not be cumbersome to enable their easy deployment and (ii) designing energy efficient algorithms for indoor positioning to maximize the battery lifetime of the mobile nodes in a localization system for disaster scenario [41].

\section{MOBILE PHONE BASED SOLUTIONS FOR DISASTER MANAGEMENT}

\section{A. Motivations}

In this section, we present the limitations of the existing solutions for disaster management. Most of the solutions for disaster management assume that in the disaster scenario, the infrastructure is in place, and affected people on the ground are already equipped with mobile device to communicate with family and rescue team. However, these solutions fail to work when a disaster occurs in areas with no cellular infrastructure prior to disaster or the infrastructure may be destroyed partially or completely. Thus, in this scenario, victims will not be able to communicate with family or rescue team and vice-versa. Several post-disaster management solutions consider the use of mobile devices by the victims to contact family and rescue team. However, such solutions are not reliable as when the existing infrastructure is damaged, the deployment of a disaster recovery network in disaster zone is usually slow, and by the time the disaster recovery network becomes operational, the battery of mobile device may get drained. Using inefficient disaster management solutions slows down the rescue efforts and causes loss of lives.

In view of this, the challenge is designing an efficient disaster management solution that enables the victims to quickly report their locations to the rescue team allowing their timely rescue. Since nowadays mobile phones are owned by almost everyone, it is but obvious for mobile phones to be an integral part of an effective disaster management solution. We are specifically interested in investigating solutions for disaster management that make use of mobile phones, not only smartphones but low-cost mobile phones as well. In this context, we discuss mobile phone disaster mode that enables mobile phones to operate in disaster mode when a disaster occurs. Disaster mode enabled mobile phone can be used both in pre-disaster and post-disaster phase. The global idea is that 
when the disaster hits an area, the mobile phones of the people on the ground should switch to disaster mode allowing to conserve the phone battery as long as possible, sending a disaster message to concerned people updating them that they are safe or need help, establishing their location quickly to the rescue team even if the infrastructure is damaged ensuring a fast rescue operation. In the following part of this section, we describe disaster mode and challenges for mobile phone disaster mode operation during a disaster. We focus on disaster management solution that use victim's mobile phone in disaster mode in order to report her accurate location allowing speedy rescue. We discuss how this solution can be enhanced with the use of efficient and accurate disaster mobile applications.

\section{B. Disaster scenarios and communication services}

Before describing the mobile phone disaster mode operation, we identify some disaster scenarios in Table III where mobile phone based disaster management should be effective and highlight the challenges in terms of communication in these disaster scenarios faced by rescue teams.

TABLE III. DISASTER SCENARIOS ( [26])

\begin{tabular}{ll}
\hline Disaster scenario & Communication challenge \\
\hline $\begin{array}{l}\text { Localised (small) urban area } \\
\text { e.g., fire in a building } \\
\text { or terrorist attack }\end{array}$ & $\begin{array}{l}\text { How to use existing local wireless networks } \\
\text { Obstacles like buildings- } \\
\text { for wireless communication }\end{array}$ \\
\hline Large scale natural disaster & How to establish communication \\
e.g., earthquake, tsunami & as infrastructure not present or damaged \\
& Lack of coverage and traffic capacity \\
\hline Indoor scenario e.g., building, & Wireless propagation hindered by walls,ceilings \\
tunnel, underground station & Limited communication options \\
& Unavailability of location apps from GNSS \\
\hline
\end{tabular}

The disaster scenarios identified in Table III require different services and capabilities from disaster communication systems [26]. Thus, a disaster communication system should provide some basic communication services as listed in Table IV with the related main features. Note that this set of services allows developing more efficient disaster applications. In the case of data connectivity and messaging services based applications, an important requirement is the bandwidth available to support the application. For example, video streaming of disaster event is not usable by rescue team if the network does not support it with an acceptable data bandwidth, as the quality of the video will not be adequate for the rescue operation.

TABLE IV. BASIC DISASTER COMMUNICATION SERVICES.

\begin{tabular}{l|l}
\hline Services & Main features \\
\hline Voice & $\begin{array}{l}\text { Must assure a certain level of voice quality } \\
\text { in disaster events with high noise e.g., explosions } \\
\text { Interactive data communication } \\
\text { Data connectivity }\end{array}$ \\
& $\begin{array}{l}\text { e.g., video streaming, query to remote servers } \\
\text { Specific Quality of Service (QoS) requirements } \\
\text { Non-interactive data communication } \\
\text { Exchange of message (text or data) }\end{array}$ \\
& $\begin{array}{l}\text { Broadcast or multicast distribution of message } \\
\text { Half-duplex communication between 2 people } \\
\text { Changing the modes e.g., from voice reception }\end{array}$ \\
Push-to-Talk & $\begin{array}{l}\text { mode to transmission mode using a button } \\
\text { Authentication, authorization, and availability }\end{array}$ \\
Security services & $\begin{array}{l}\text { Determine location of victims/rescue team in the field } \\
\text { Provided by GNSS like GPS }\end{array}$ \\
\hline
\end{tabular}

\section{Disaster situation aware protocols for mobile devices}

Integrating context aware computing with mobile devices enable them to adapt and react to dynamic changes in the environment. This concept is used in [42] to design a context aware ad hoc network for effective crowd disaster mitigation by issuing an alert to prevent a stampede in the crowded area. We are interested in designing as well as adapting existing protocols and algorithms which we term as Disaster Aware Protocols (DAP) taking into account the disaster situation allowing mobile devices to be effective in a disaster scenario. In a disaster scenario, in the absence (or partial presence) of an infrastructure, a mobile device should be able to operate in a disaster mode serving as a lifeline for the common people on the ground. Disaster situation aware protocols or algorithms for mobile devices should feature communication mode switching scheme in which information such as amount of remaining battery, mobility (mobile phone's movement), and number of neighboring mobile devices is used by the mobile phone to decide the apt communication mode [24]. In this context, we are interested in studying if $5 \mathrm{G}$ networks can feature mode selection schemes that manage the switching between different communication modes, such as normal mode and disaster mode. For example, in 5G, the use of ad-hoc D2D communications in the disaster mode can offer minimal connectivity during disaster.

One recent work that focus on enabling smartphone disaster mode is by Geoffery et al. [43]. The project [43] focuses on enabling smartphone disaster mode, however we are interested in a solution for high-end as well as low-end mobile phones since a large number of population in developing countries such as Nepal that was affected by earthquakes recently, might not have access to expensive smartphones. A universal disaster communication solution should be supported by mobile phones with varied operating systems (iOs, Android, Windows, etc.). The mobile phone should be equipped with the minimum computational capacity enough for automatically switching to and operating in disaster mode (e.g., D2D communications mode) in disaster areas with damaged infrastructures [24]. In the next part, we describe disaster mode and challenges for mobile phone disaster mode operation during disaster.

\section{Mobile phone disaster mode}

With the proliferation of mobile devices (mobile phones and tablets) in our lives and almost everyone possessing a mobile phone these days, the mobile phone is a potential device in the event of a disaster scenario to be able to help us connect with family and friends, locate resources, navigate to a safer location and help others. In addition, smartphones can use their integrated sensors to help allocate scarce resources to the most affected people by collecting data enabling disaster relief teams to comprehend the unraveling situation in the disaster zone.

However, due to the challenges of energy-management and connectivity smartphones that are currently available are not well equipped to operate efficiently during disaster. Often, disaster victims are left helpless with poor or no connectivity and mobile phones running out of battery quickly. Table $\mathrm{V}$ highlights the key challenges for mobile phone disaster mode [44]. The normal mode of operation for smartphones assume that energy is ample and high-speed mobile data and $\mathrm{WiFi}$ 
TABLE V. CHALlENGES FOR SMARTPHONE DISASTER MODE OPERATION.

\begin{tabular}{l|l|l}
\hline Challenges / Features & Normal mode & Disaster mode \\
\hline Battery life / Power & Plentiful & Scarce (extreme) \\
Internet connectivity & Common & Rare \\
User behavior & Typical & Atypical \\
Dependency & Essential & Critical \\
\hline
\end{tabular}

TABLE VI. MAIN FEATURES OF MOBILE DISASTER MODE BEFORE AND DURING DISASTER.

\begin{tabular}{l}
\hline Pre-disaster phase (Before disaster) \\
\hline Identify vulnerable people (most prone to the impacts of disaster) \\
Help allocate scarce disaster resources \\
\hline Post-disaster phase (During disaster) \\
\hline Remain useful (mobile to be remain useful) \\
Self-organize into a per-disaster social group to improve survivability \\
Help authorities track unfolding events \\
\hline
\end{tabular}

networks are almost always available. While during a disaster, such assumptions fail; power is scarce (the phone battery may need to last for several days) and networks are down or overloaded (partially or fully damaged).

Geoffery et al. focus on developing Android platform support for a disaster mode allowing smartphones to prepare for and operate efficiently during disaster. In the pre-disaster phase i.e., when a disaster is impending, disaster mode helps users get ready (to move to safer location), by preparing their phone and collecting data to identify vulnerable people, most prone to the impact of disaster. While in the postdisaster phase, i.e., when a disaster hits, disaster mode allows extreme energy management, novel user interfaces, and new communication patterns, all working together to retain vital functionality and at the same time maximizing battery life. Table VI shows the main features of mobile disaster mode in pre- and post-disaster phase i.e., before the disaster strikes (while disaster is impending) and during the disaster [44].

1) Possible scenario with disaster mode enabled mobile phone: We describe here a possible scenario with disaster mode enabled mobile phone in pre-disaster phase and during the disaster [43].

Pre-disaster phase (before disaster): In disaster-prone regions, users should be able to download and install a disaster management application on their phones either through public service announcements or emergency messages sent directly to their phones. This app would start gathering data to locate and identify vulnerable people and based on that data, they will be assigned higher priority of rescue operation. Using the app, local people on the ground would be able to connect with their families through, e.g., social networks to update their safety status and also to make decisions about moving to a safer location. The information gathered by the disaster app during pre-disaster phase (i.e., right before the disaster like earthquake hits a region), would be of use in the post-disaster phase. As an example, setting up connectivity maps before the disaster can help locate and identify areas that may lose connectivity and detect failures after the disaster hits.

Post-disaster phase (during disaster): Once disaster hits a region, the mobile phone will turn on its disaster mode and will start operating in the disaster mode. The disaster mode allows extreme energy-savings through intelligent management of device resources and apps in addition to novel low-power

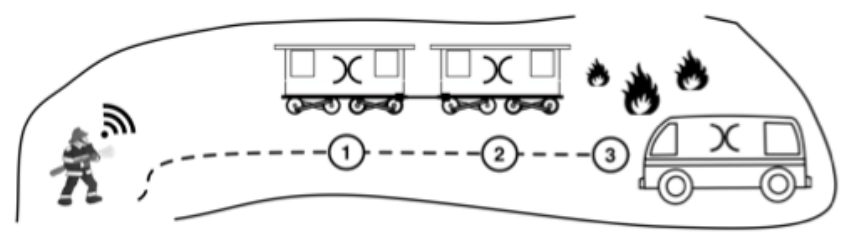

Fig. 3. Beacons placed inside train wagons and bus [45].

user interfaces. Moreover, disaster mode prevents the rapid energy drain out due to the loss of WiFi connectivity and potential overloads of the cellular networks. In disaster mode, low-power localization and resource discovery approaches can be used to minimize energy consumption while enabling users to navigate out of the disaster hit area and connect with family. Furthermore, peer-to-peer networking technologies can be used to spread rescue information across disconnected devices and also help victims form support groups and move to safety.

\section{E. Help Beacons system for smartphone}

A recent work by Amro Al-Akkad et al. [45] introduces "Help Beacons" system, a lightweight S.O.S. system for smartphones that enables ad-hoc communication between first responders and victims in disaster where existing network infrastructure has become disrupted. The proposed system enables offline communications through the detection of emergency beacons by exploiting the Wi-Fi SSID and allows for short-lived ad-hoc connections among neighboring devices by piggybacking on the WiFi handshake. The Help Beacons system uses Wi-Fi SSIDs as beacons to seek help, to offer resources, or to reveal locations during disaster. The formation of an emergency beacon defines a "I'm here" signal, that can help responders in locating missing persons and re-establishing communication. Figure 3 shows beacons placed inside two train wagons and one bus.

The Help Beacons system consists of two mobile applications; victim application and first responder application. The first application allows the broadcasting of distress signals using Wi-Fi SSIDs. It allows the users to generate an emergency beacon by putting short messages inside the SSID of a Wi-Fi network. While the second application allows first responders to locate the emergency Beacons and thereby, trace the people broadcasting the distress signals. A Beacon is created once the user pushes the S.O.S. button and consequently, a WiFi network is deployed. The responder application detects radio signals to look for Beacons. The discovery of Beacons is triggered by the user by pushing the Search button. The system consists of two devices namely Beacon device and Seeker device. First, a Beacon device sets up a Wi-Fi network that uses a specific string of characters inside the SSID to advertise itself as a Beacon. The Beacon device runs a DHCP server to provide an IP address to the Seeker device, and listens to a particular socket address to exchange information, when the Seeker device connects to it. There is a 30s timeout for the responder application to connect to available Beacons and handle the problem of fake Beacons. A failure in connecting to a Beacon leads to blacklisting of the corresponding SSID for $1 \mathrm{~min}$. 
Help Beacons system uses Wi-Fi in Infrastructure Mode thereby addressing the issues of limited transmission range and initial pairing of devices. The authors claim that their system is different from existing platforms for communication in disaster situations in that it does not depend on an existing network infrastructure and operates on off-the-shelf, commercially available smartphones. The mobile applications are developed for Android. One shortcoming of this system is that configuration of the application is not efficient for a first responder to be able to use it with ease during disaster. Another issue is that during disaster how users can install the victim application on their mobiles.

\section{OPEN RESEARCH CHALLENGES}

In section IV, we identified some research challenges for future wireless communication technologies during disaster to explore further. Primary challenges are related to energy efficiency, connectivity, QoS, availability or resilience, and interoperability. Note that the selection of the technology and associated research challenges depend on the particular disaster scenario (e.g., nature disaster scenario or crisis in urban area scenario) or applications. In this paper, we discussed the potential use of disaster mode enabled mobile phone in disaster scenario. However, we are still far from being able to use the smartphones to their full potential during disaster. We identify several research issues that must be investigated to enable mobile phones operate efficiently during disaster which are as follows [44]; extreme energy management, low-power localization and navigation, peer-to-peer communication, improve resilience models, and quantifying social connectedness. Some of the main challenges are discussed below [43].

Battery life: Specific disaster environment-related features such as loss of power, navigation, and poor connectivity further decrease the already poor battery life of mobile phone. Extreme energy management is the most important feature in disaster mode to prevent phones from energy drain out. New energy management approaches need to be investigated to maximize battery life through efficient resource discovery, energy-efficient localization techniques, and low-power user interfaces. Moreover, the mobile platform should be improvised to enhance user control over the apps running during the disaster while ensuring that background energy consumption is limited.

Internet connectivity: Wireless (WiFi APs) network infrastructure might be partially or fully damaged during the disaster. Mobile data and voice networks may be operational, but are probable to be overloaded. Further studies are required to understand the impact of disasters on cellular network performance to examine the novel connectivity primitives that are apt to consider in mobile phone disaster mode and their implementation.

Vulnerability, resilience and reporting: New methods should be explored to use the data gathered (e.g., location information, degree of mobility) from mobile phones to measure vulnerability (and resilience) at macro (community) as well as micro (individual) levels. Observing the area of an individual and traveling pattern can help in determining her mobility rate, transportation access, and familiarity with areas and paths she may require to take to escape the disaster. Another challenge is how to use mobile phones to determine the disaster survivability e.g., for an individual, through their level of connectivity with friends and social network.

\section{CONCLUSION}

Recent disasters have brought the focus on the ever growing significance of disaster management systems and networks. In this paper, we have focused on how disaster management can benefit from recent advances in wireless communication technologies and protocols, especially mobile technologies and devices. We have surveyed various potential emerging wireless communication technologies such as 5G, D2D, LTE, and software defined radio for disaster situations. In particular, we have focused on the use of ubiquitous mobile devices and applications in disaster situations.

\section{REFERENCES}

[1] Quartz. India. http://qz.com/406562/how-social-media-is-helping-nepalrebuild-after-two-big-earthquakes-2/, 2015.

[2] "Safety Check." https://www.facebook.com/about/safetycheck/, 2015.

[3] S. Krishnamoorthy and A. Agrawala, "M-urgency: a next generation, context-aware public safety application." in Proceedings of the 13th International Conference on Human Computer Interaction with Mobile Devices and Services. ACM, 2011, pp. 647-652.

[4] "NowForce." http://www.nowforce.com/.

[5] "SafeCity Get live information from the society," http://safecity.nl/.

[6] "Ushahidi Crowdmap," http://www.ushahidi.com/.

[7] T. Hossmann, F. Legendre, P. Carta, P. Gunningberg, and C. Rohner, "Twitter in disaster mode," ExtremeCom2011, 2011.

[8] A. Martín-Campillo, R. Martí, E. Yoneki, and J. Crowcroft, "Electronic triage tag and opportunistic networks in disasters," in Proceedings of the Special Workshop on Internet and Disasters. ACM, 2011, p. 6.

[9] Index, Cisco Visual Networking, "Global mobile data traffic forecast update, 2014-2019 white paper," 2015.

[10] “Open Garden Inc," https://opengarden.com/.

[11] D. Camps-Mur, A. Garcia-Saavedra, and P. Serrano, "Device-to-device communications with wi-fi direct: overview and experimentation," Wireless Communications, IEEE, vol. 20, no. 3, pp. 96-104, 2013.

[12] S. Lu, S. Shere, Y. Liu, and Y. Liu, "Device discovery and connection establishment approach using ad-hoc wi-fi for opportunistic networks," in Pervasive Computing and Communications Workshops (PERCOM Workshops), 2011 IEEE International Conference on. IEEE, 2011, pp. 461-466.

[13] S. Trifunovic, B. Distl, D. Schatzmann, and F. Legendre, "Wifi-opp: ad-hoc-less opportunistic networking," in Proceedings of the 6th ACM workshop on Challenged networks. ACM, 2011, pp. 37-42.

[14] R. G. L. Narayanan and O. C. Ibe, "A joint network for disaster recovery and search and rescue operations," Computer Networks, vol. 56, no. 14, pp. 3347-3373, 2012.

[15] T. Doumi et al., "LTE for public safety networks," Communications Magazine, IEEE, vol. 51, no. 2, pp. 106-112, 2013.

[16] G. Fettweis and S. Alamouti, "5G: Personal mobile internet beyond what cellular did to telephony," Communications Magazine, IEEE, vol. 52, no. 2, pp. 140-145, February 2014.

[17] L. Militano et al., "Efficient Spectrum Management Exploiting D2D Communication in 5G Systems," arXiv preprint arXiv:1504.06813, 2015 .

[18] A. Asadi, Q. Wang, and V. Mancuso, "A survey on device-to-device communication in cellular networks," Communications Surveys \& Tutorials, IEEE, vol. 16, no. 4, pp. 1801-1819, 2014.

[19] P. Pahlevani et al., "Novel concepts for device-to-device communication using network coding," Communications Magazine, IEEE, vol. 52, no. 4, pp. 32-39, 2014. 
[20] G. Fodor, S. Parkvall, S. Sorrentino, P. Wallentin, Q. Lu, and N. Brahmi, "Device-to-device communications for national security and public safety," Access, IEEE, vol. 2, pp. 1510-1520, 2014.

[21] K. Gomez et al., "A Comparative Study of Scheduling Disciplines in 5G Systems for Emergency Communications," in $5 G$ for Ubiquitous Connectivity (5GU), 2014 1st International Conference on. IEEE, 2014, pp. 40-45.

[22] M. Hunukumbure, T. Moulsley, A. Oyawoye, S. Vadgama, and M. Wilson, "D2D for Energy Efficient Communications in Disaster and Emergency Situations," in Software, Telecommunications and Computer Networks (SoftCOM), 2013 21st International Conference on. IEEE, 2013, pp. 1-5.

[23] A. Orsino, L. Militano, G. Araniti, A. Molinaro, and A. Iera, "Efficient Data Uploading Supported by D2D Communications in LTE-A Systems," arXiv preprint arXiv:1503.09076, 2015.

[24] H. Nishiyama, M. Ito, and N. Kato, "Relay-by-smartphone: realizing multihop device-to-device communications," Communications Magazine, IEEE, vol. 52, no. 4, pp. 56-65, 2014

[25] O. Bay, "5G in 2020 Will Be Rare; Over 100 Million Subscribers by 2025," https://www.abiresearch.com/press/5g-in-2020-will-be-rareover-100-million-subscribe/, 2014.

[26] G. Baldini, S. Karanasios, D. Allen, and F. Vergari, "Survey of wireless communication technologies for public safety," Communications Surveys \& Tutorials, IEEE, vol. 16, no. 2, pp. 619-641, 2014.

[27] R. Ferrús, O. Sallent, G. Baldini, and L. Goratti, "LTE: the technology driver for future public safety communications." IEEE Communications Magazine, vol. 51, no. 10, pp. 154-161, 2013.

[28] M. Peng, D. Liang, Y. Wei, J. Li, and H.-H. Chen, "Self-configuration and self-optimization in LTE-advanced heterogeneous networks," Communications Magazine, IEEE, vol. 51, no. 5, pp. 36-45, 2013.

[29] M. Panizza et al., "Feasibility study of a SDR-based reconfigurable terminal for emergency applications," in Aerospace Conference, 2011 IEEE. IEEE, 2011, pp. 1-18.

[30] G. Baldini et al., "The EULER project: application of software defined radio in joint security operations," Communications Magazine, IEEE, vol. 49, no. 10, pp. 55-62, 2011.

[31] J. Mitola and G. Q. Maguire Jr, "Cognitive radio: making software radios more personal," Personal Communications, IEEE, vol. 6, no. 4 pp. 13-18, 1999.

[32] T. L. Doumi, "Spectrum considerations for public safety in the united states," Communications Magazine, IEEE, vol. 44, no. 1, pp. 30-37, 2006

[33] A. Gorcin and H. Arslan, "Public safety and emergency case communications: Opportunities from the aspect of cognitive radio," in New Frontiers in Dynamic Spectrum Access Networks, 2008. DySPAN 2008. 3rd IEEE Symposium on. IEEE, 2008, pp. 1-10.

[34] T. F. Rahman and C. Sacchi, "Opportunistic radio access techniques for emergency communications: Preliminary analysis and results," in Satellite Telecommunications (ESTEL), 2012 IEEE First AESS European Conference on. IEEE, 2012, pp. 1-7.

[35] N. Jesuale, "Lights and sirens broadband How spectrum pooling, cognitive radio, and dynamic prioritization modeling can empower emergency communications, restore sanity and save billions," in New Frontiers in Dynamic Spectrum Access Networks (DySPAN), 2011 IEEE Symposium on. IEEE, 2011, pp. 467-475.

[36] J. Wang, M. Ghosh, and K. Challapali, "Emerging cognitive radio applications: A survey," Communications Magazine, IEEE, vol. 49, no. 3, pp. 74-81, 2011.

[37] S. Ingram, D. Harmer, and M. Quinlan, "Ultrawideband indoor positioning systems and their use in emergencies," in Position Location and Navigation Symposium, 2004. PLANS 2004. IEEE, 2004, pp. 706-715.

[38] B. Alavi and K. Pahlavan, "Bandwidth effect on distance error modeling for indoor geolocation," in Personal, Indoor and Mobile Radio Communications, 2003. PIMRC 2003. 14th IEEE Proceedings on, vol. 3 IEEE, 2003, pp. 2198-2202.

[39] O. Krejcar, "Using of mobile device localization for several types of applications in intelligent crisis management," in GCC Conference \& Exhibition, 2009 5th IEEE. IEEE, 2009, pp. 1-5.

[40] D. Borio, C. O'Driscoll, and J. Fortuny-Guasch, "Pulsed pseudolite signal effects on non-participating GNSS receivers," in Indoor Posi- tioning and Indoor Navigation (IPIN), 2011 International Conference on. IEEE, 2011, pp. 1-6.

[41] J. J. Robles, S. Tromer, M. Quiroga, and R. Lehnert, "A low-power scheme for localization in wireless sensor networks," in Networked Services and Applications-Engineering, Control and Management. Springer, 2010, pp. 259-262.

[42] M. V. Ramesh et al., "Context aware ad hoc network for mitigation of crowd disasters," Ad Hoc Networks, vol. 18, pp. 55-70, 2014.

[43] "Smartphone Disaster Mode," http://smartamerica.org/teams/smartphonedisaster-mode/.

[44] "Smartphone Disaster Mode," http://smartamerica.org/wpcontent/uploads/2014/05/disastermode.pdf/, 2014.

[45] A. Al-Akkad et al., "Help Beacons: Design and Evaluation of an AdHoc Lightweight SOS System for Smartphones," in Proceedings of the SIGCHI Conference on Human Factors in Computing Systems. ACM, 2014, pp. 1485-1494. 